\title{
Design and Characterization of an RF Plasma Cleaner
}

\author{
G.P. Canal ${ }^{\ddagger}$, H. Luna”, L.F. Ruchko\& and R.M.O. Galvão ${ }^{\ddagger}$. \\ ${ }^{\ddagger}$ Centro Brasileiro de Pesquisas Físicas. Laboratório de Plasmas Aplicados. Rua Xavier Sigaud 150, 22.290-180. Rio de Janeiro, Brazil \\ ${ }^{\#}$ Instituto de Física, Universidade Federal do Rio de Janeiro, \\ Cx. Postal 68.528, Rio de Janeiro, RJ 21.941-972, Brazil and \\ ${ }^{\&}$ Instituto de Física, Universidade de São Paulo,São Paulo, SP 05.508-090, Brazil
}

(Received on 30 December, 2009)

\begin{abstract}
The design and characterization of an innovative device for plasma cleaning, based upon a non-conventional radio-frequency discharge, is described. The RF fields are produced by an antenna placed inside the metallic vacuum chamber. Theoretical models are described to calculate both the electro-magnetic field structure and the spatial impurity distribution, due to erosion of the antenna. The electron energy distribution function is determined with a standard RF-filtered electrostatic probe; it is found that the plasma is well described by a Druyvesteyn energy distribution function.
\end{abstract}

Keywords: RF discharge, plasma cleaner, Langmuir probe

\section{INTRODUCTION}

Low-temperature plasmas are widely employed in technological micro-electronics and nanotechnology processes [1], either in the fabrication scheme or as an auxiliary tool, for pre or post surface treatment [2-4]. In particular, proper preparation of the samples is absolutely essential in electron microscopy, to avoid artifacts or contaminants, usually hydrocarbons originated in their manipulation.

Among the several methods for preparation of samples for SEM (scanning electron microscopy) and TEM (tunneling electron microscopy), one commonly used is the Plasma Cleaner. This is usually a small vacuum chamber in which the plasma is produced by an inductive-coupled RF discharge, with the antenna placed outside the vacuum chamber [5]. This scheme avoids the plasma contamination observed in capacitively coupled discharges, widely used in the electronic industry, due to erosion of the electrodes inside the vacuum chamber [6].

Although the plasma cleaners are commercially available, they are usually assembled as a integrated and compact device, optimized for the cleaning process. This makes it difficult the access for plasma diagnostics. Only external parameters, such as RF power, pressure, and type of working gas, are available to the experimentalist to control the process. When there is interest in using the plasma not only as an auxiliary tool, but also as the object of study, to properly investigate the relevant physical mechanisms involved in the plasma-surface interaction, a more flexible assembly is required.

Another feature of the commercially available plasma cleaners is that the antenna is placed outside the vacuum chamber. This imposes the use of a dielectric window, either a quartz cylindrical tube or a plate, so that the vacuum chamber is not entirely metallic. For the vacuum conditions of conventional plasma cleaners, the presence of the dielectric window does not spoil the quality of the cleaning process. However, when one is interested in investigating the performance of low pressure discharges, for special applications, or non-standard plasma mechanisms, such as collisionless RF power absorption, a metallic wall is required to guarantee proper vacuum conditions and unambiguous results.

In this work we present the design and characterization of an inductively coupled plasma cleaner with the antenna placed inside the vacuum chamber. The antenna is made of circular loops concentric with the cylindrical metallic chamber. In this configuration, the vacuum electro-magnetic field configuration can be analytically calculated, facilitating the modeling of the discharge. This is discussed in the next section, together with the model to calculate the spatial distribution of the impurities generated by sputtering of the antenna. As found in the literature[5], we show that the level of impurities is negligible at the position where the substrate is placed. The experimental set up is presented in Section 3 and the characterization of the discharge in Section 4. One important result obtained in this work is that the electron energy distribution function is not Maxwellian, but better represented by a Druyvesteyn function, within the working pressure range of $3.0 \times 10^{-2}$ to $1.5 \times 10^{-1}$ mbar.

\section{THEORETICAL MODEL}

\subsection{Electromagnetic Fields}

In order to study the electro-magnetic field inside the chamber, we developed a theoretical model in which the reactor is regarded as a perfectly conducting cylinder of radius $b$ and height $H$. The antenna used for generation of the RF field is modeled by a current loop of radius $a$ positioned at a height $h$ inside the reactor (Fig. 1).

The distribution of the electro-magnetic field inside the vacuum chamber is obtained by solving the wave equation for the vector potential $[8,9]$,

$$
\nabla^{2} \vec{A}-\frac{1}{c^{2}} \frac{\partial^{2} \vec{A}}{\partial t^{2}}=-\mu_{0} \vec{J}
$$

with the current density $\vec{J}$ modeled by a loop current given by

$$
\vec{J}(r, z, t)=I_{0} e^{i \omega t} \delta(r-a) \delta(z-h) \widehat{e}_{\varphi} .
$$

where $\omega$ is the angular frequency and $I_{0}$ the amplitude of the RF current.

Substituting equation 2 into equation 1, considering that the vector potential has only the $\varphi$ component, and using the expression for the Laplacian operator in cylindrical coordi- 


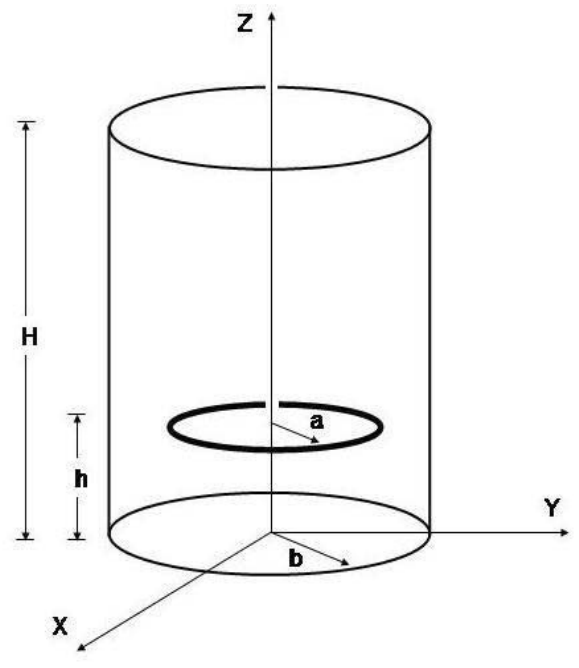

FIG. 1: Schematic drawing of the theoretical model for the plasma reactor

nates, we obtain

$$
\begin{aligned}
& \frac{1}{r} \frac{\partial}{\partial r}\left(r \frac{\partial A_{\varphi}}{\partial r}\right)+\frac{\partial^{2} A_{\varphi}}{\partial z^{2}}+\left(k^{2}-\frac{1}{r^{2}}\right) A_{\varphi} \\
& =-\mu_{0} I_{0} \delta(r-a) \delta(z-h)
\end{aligned}
$$

where $c$ is the velocity of light and $k=\omega / c$ is the wave number.

Solving the associated homogeneous equation by separation of variables and applying the boundary condition on the fields at the conducting wall, we obtain the eigenfunctions of the problem

$$
\psi_{m, n}(r, z)=J_{1}\left(j_{1, n} \frac{r}{b}\right) \operatorname{sen}\left(\frac{m \pi z}{H}\right)
$$

with their respective eigenvalues given by

$$
\lambda_{m, n}^{2}=\left(\frac{j_{1, n}}{b}\right)^{2}+\left(\frac{m \pi}{H}\right)^{2} .
$$

where $j_{v, n}$ is the $n$th root of the Bessel function $J_{v}(x)$. Developing the vector potential on the basis of the eigenfunctions

$$
A_{\varphi}(r, z)=\sum_{m=1}^{\infty} \sum_{n=1}^{\infty} a_{m, n} \psi_{m, n}(r, z)
$$

and substituting it into equation 3 , to take into account the source term, the $a_{m, n}$ coefficients are obtained

$$
a_{m, n}=\frac{4 a \mu_{0} I_{0} \operatorname{sen}\left(\frac{m \pi h}{H}\right) J_{1}\left(j_{1, n} \frac{a}{b}\right)}{H b^{2}\left[\left(\frac{j_{1, n}}{b}\right)^{2}+\left(\frac{m \pi}{H}\right)^{2}-k^{2}\right]\left[J_{2}\left(j_{1, n}\right)\right]^{2}} .
$$

Using the known relation between the vector potential and fields, it is possible to obtain the electric and magnetic fields inside the chamber,

$$
\vec{E}(r, z, t)=\sum_{m=1}^{\infty} \sum_{n=1}^{\infty} \omega a_{m, n} J_{1}\left(j_{1, n} \frac{r}{b}\right) \sin \left(\frac{m \pi z}{H}\right) \sin (\omega t) \widehat{e}_{\varphi},
$$

the radial component of magnetic field,

$$
B_{r}(r, z, t)=-\frac{\pi}{H} \sum_{m=1}^{\infty} \sum_{n=1}^{\infty} a_{m, n} m J_{1}\left(j_{1, n} \frac{r}{b}\right) \cos \left(\frac{m \pi z}{H}\right) \cos (\omega t)
$$

and its z-component

$B_{z}(r, z, t)=\sum_{m=1}^{\infty} \sum_{n=1}^{\infty} a_{m, n} \frac{j_{1, n}}{b} J_{0}\left(j_{1, n} \frac{r}{b}\right) \sin \left(\frac{m \pi z}{H}\right) \cos (\omega t)$.

The fields of an antenna with more than one current loop can be obtained simply by linear superposition. The currents induced at the wall of the vacuum chamber can then be obtained from the boundary condition for the tangential component of the magnetic field. In the actual device, we find it more adequate to employ three current loops. The corresponding magnetic field lines and profiles of the currents in the wall of the vacuum chamber are shown in figure 2 .

Finally, using equations 9 and 10 is possible to find the inductance of the system,

$$
L=\frac{1}{\mu_{0} I_{0}^{2}} \int\left(B_{r}^{2}+B_{z}^{2}\right) d V
$$

\subsection{Diffusion of Impurities}

Because the antenna is placed inside the vacuum chamber, contamination of the samples, due to the material eroded from the antenna, might occur. Yamashita [5] has shown experimentally that in a similar system the sample contamination could be disregarded. To address this problem, we developed a theoretical model to study the spatial distribution of the impurity density in the plasma, due to erosion of the antenna. The corresponding diffusion equation

$$
\nabla^{2} n-\frac{1}{D} \frac{\partial n}{\partial t}=-\frac{F}{D}
$$

is solved inside the vacuum chamber, with the source term $F$ given by the sputtering rate at the antenna. In this equation, $n$ is the impurity density and $D$ the diffusion coefficient, assumed to be constant.

To proceed, we specify the source term at the antenna, i.e.,

$$
F(r, z, t)=F_{0} \delta(r-a) \delta(z-h),
$$

where $F_{0}$ is the particles sputtering rate.

To compare the density of particles eroded from the antenna $n$, calculated using this model, with neutral gas density $n_{n}$ it is necessary to know the values of the free diffusion coefficient $D$ and the particle sputtering rate $F_{0}$. For a simple estimate we can assume the diffusion coefficient constant and given by

$$
D=\frac{k_{B} T_{\text {sputt }}}{M_{\text {sputt }} \mathrm{v}_{c}}
$$

where $v_{c}, M_{\text {sputt }}$, and $T_{\text {sputt }}$ are the collision frequency, particle mass, and temperature of eroded particles from the antenna, respectively, and

$$
F_{0}=\int \vec{\Gamma}_{\text {sputt }} \cdot \hat{n} d S
$$



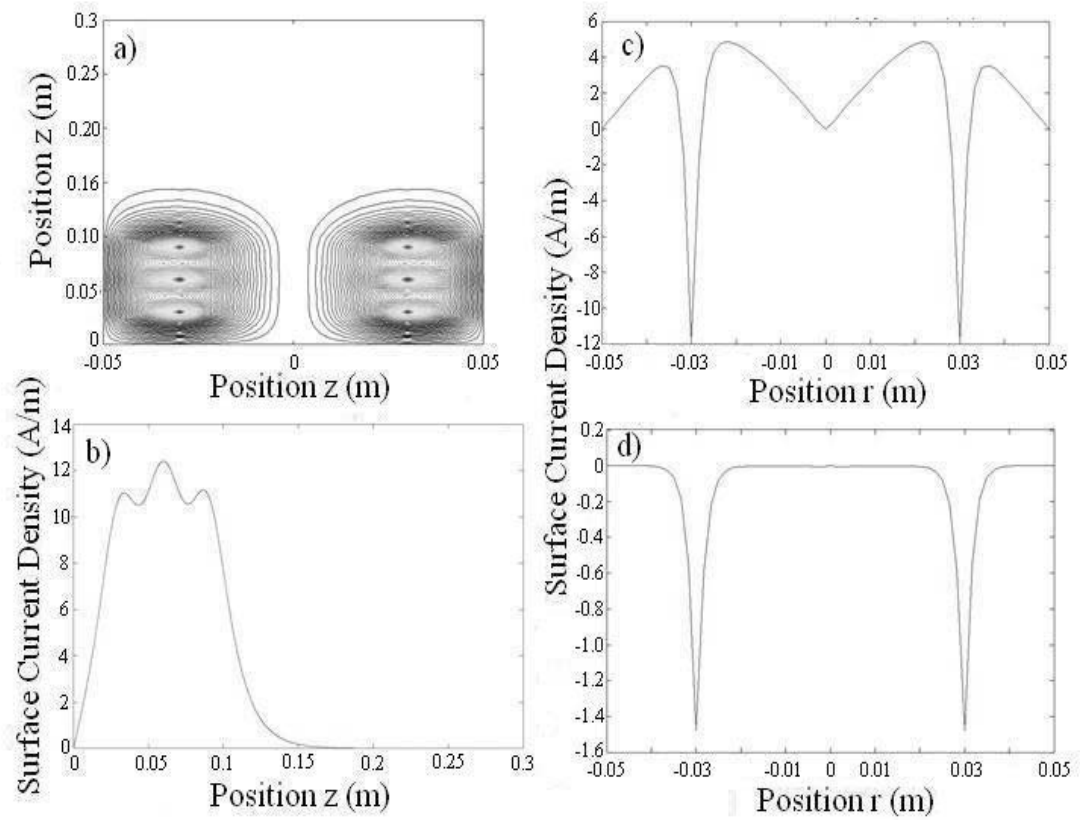

FIG. 2: a) Magnetic field lines and surface current density b) on the wall, c) on the base and d) on the top of the chamber for three loop antenna

where the flow of sputtered particles from the antenna, $\vec{\Gamma}_{\text {sputt }}$, is integrated over its surface. We also assume that the sputtered particles from the antenna are in thermal equilibrium with the neutral gas, i.e., at room temperature.

As can be seen in reference [7], for a pressure near $4.0 \times$ $10^{-2}$ mbar, we have that $v_{c}=\omega$. Therefore, $v_{c}=1.36 \times 10^{7}$ Hz. The mainly particles eroded from the antenna correspond to iron atoms, which has $M_{\text {sputt }}=9.4 \times 10^{-26} \mathrm{~kg}$, so that we find the free diffusion coefficient $D \approx 1.0 \times 10^{-3} \mathrm{~m}^{2} \mathrm{~s}^{-1}$.

To determine $F_{0}$, we assume that the flow of ions to be uniform on the whole surface of the antenna; using the value of the surface area approximately $S=1 \times 10^{-4} \mathrm{~m}^{2}$, we can estimate the value of $F_{0}$ using the experimental results obtained in this work.

By considering the ion flow given by,

$$
\Gamma_{i}=n_{i} v_{B}
$$

where $n_{i}$ is the ion density and $v_{B}$ the Bohm velocity, given by

$$
v_{B}=\sqrt{\frac{k_{B} T_{e}}{M_{i}}},
$$

and assume that the flow of sputtered particles is given by

$$
\Gamma_{\text {sputt }}=\gamma \Gamma_{i},
$$

where $\gamma$ is the sputtering yield; its value for iron and to the range of energies reached in the actual experimental conditions is around $10^{-3}$.

Using the values obtained for the pressure of $4.0 \times 10^{-2}$ mbar near to the antenna, $n_{i}=3 \times 10^{15} \mathrm{~m}^{-3}, T_{e}=4 \mathrm{eV}$ and argon mass $M_{i}=M_{n}=6.68 \times 10^{-26} \mathrm{~kg}$, we find that $F_{0}$ $\approx 1,0 \times 10^{12} s^{-1}$.

The diffusion equation can be solved by introducing separation of variables and by applying the proper boundary conditions. Therefore the eigenfunction of the problem is obtained

$$
\varphi_{m, n}(r, z)=J_{0}\left(j_{0, n} \frac{r}{b}\right) \sin \left(\frac{m \pi z}{H}\right)
$$

with their eigenvalues being given by

$$
\xi_{m, n}^{2}=\left(\frac{j_{0, n}}{b}\right)^{2}+\left(\frac{m \pi}{H}\right)^{2} .
$$

Expanding $n(\vec{r}, t)$ and $F(\vec{r}, t)$ on the basis of eigenfunctions

$$
n(\vec{r}, t)=\sum_{n=1}^{\infty} \sum_{m=1}^{\infty} a_{m, n}(t) \varphi_{m, n}(\vec{r})
$$

and

$$
F(\vec{r}, t)=\sum_{n=1}^{\infty} \sum_{m=1}^{\infty} c_{m, n}(t) \varphi_{m, n}(\vec{r}),
$$

and substituting back into equation 12 , the coefficients $a_{m, n}(t)$ are obtained

$$
a_{m, n}(t)=e^{-D \xi_{m, n} t} \int_{0}^{t} c_{m, n}(\tau) e^{D \xi_{m, n} \tau} d \tau .
$$

To find $a_{m, n}(t)$ we need, therefore, to determine the $c_{m, n}$ coefficient; inverting equation 22 we have 


$$
c_{m, n}(t)=\frac{4}{H b^{2}\left[J_{1}\left(j_{0, n}\right)\right]^{2}} \int_{0}^{b} \int_{0}^{H} r J_{0}\left(j_{0, n} \frac{r}{b}\right) \sin \left(\frac{m \pi z}{H}\right) F(r, z, t) d r d z
$$

Finally using the source term, we find the density of impurities inside the reactor, for $t>0$,

$$
n(r, z, t)=\sum_{n=1}^{\infty} \sum_{m=1}^{\infty} a_{m, n}(t) J_{0}\left(j_{0, n} \frac{r}{b}\right) \sin \left(\frac{m \pi z}{H}\right)
$$

where $a_{m, n}$ are the coefficients given by

$$
a_{m, n}(t)=\frac{4 a F_{0}\left[1-e^{-D \xi_{m, n} t}\right]}{H b^{2} D \xi_{m, n}\left[J_{1}\left(j_{0, n}\right)\right]^{2}} J_{0}\left(j_{0, n} \frac{a}{b}\right) \sin \left(\frac{m \pi h}{H}\right) .
$$

Using Fick's law and taking into account that $\Gamma_{z}$ is the only component of the particle flux $\vec{\Gamma}_{\text {sputt }}$ that contributes to the flow toward the sample, we find that at the position of the sample, $r=0$ and $z=z_{0}$,

$$
\Gamma_{z}=-\frac{\pi}{H} \sum_{n=1}^{\infty} \sum_{m=1}^{\infty} m D a_{m, n} \cos \left(\frac{m \pi z_{0}}{H}\right) .
$$

In the region between $z_{0}=20$ and $z_{0}=30 \mathrm{~cm}$, which is the region where the sample is normally placed, we have that the ratio $R=n_{\text {sputt }} / n_{n}$, for pressures of the order of $\mathrm{p}=1,0 \times 10^{-2} \mathrm{mbar}$, is always smaller or of the order of $R=1,0 \times 10^{-10}$, i.e., the quantity of sputtered particles from the antenna at the sample can be considered negligible. The corresponding flux of sputtered particles on the sample is $\Gamma_{\text {sputt }}=1,0 \times 10^{8} \mathrm{~m}^{-2} \mathrm{~s}^{-1}$.

To compare this with the flux of the background neutral particle, we take their mean velocity given by

$$
\left\langle v_{n}\right\rangle=\sqrt{\frac{8 k_{B} T_{n}}{\pi M_{n}}}
$$

Substituting the temperature $\left(T_{n}=300 \mathrm{~K}\right)$ and the mass of the neutral $\left(M_{n}=6,68 \times 10^{-26} \mathrm{~kg}\right.$ for Argon) we obtain the mean velocity as $\left\langle v_{n}\right\rangle=397.3 \mathrm{~m} / \mathrm{s}$. Then, using the equation

$$
\Gamma_{n}=\frac{1}{4} n_{n}\left\langle v_{n}\right\rangle
$$

where $n_{n}\left[m^{-3}\right]=2,42 \times 10^{22} p, p$ being the pressure of the gas in mbar, we obtain $\Gamma_{n}=2,4 \times 10^{22} \mathrm{~m}^{-2} \mathrm{~s}^{-1}$, for $\mathrm{p}=$ $4,0 \times 10^{-2}$ mbar. Using the equation 29 to estimate the flow of ions and electrons over the sample and using the results obtained in this work, we find that $\Gamma_{e}=4,0 \times 10^{17}$ $\mathrm{m}^{-2} \mathrm{~s}^{-1}$ and $\Gamma_{i}=1,5 \times 10^{17} \mathrm{~m}^{-2} \mathrm{~s}^{-1}$. Therefore, we have that $\Gamma_{\text {sputt }} \ll \Gamma_{e} \approx \Gamma_{i} \ll \Gamma_{n}$. From this results we conclude that the contamination of the sample by sputtered material from the antenna is not substantial, confirming the experimental result obtained by Yamashita[5].

For a steady state condition, we take $t \rightarrow \infty$ and plot the density of impurities as function of position inside the reactor, which is shown in figure 3 . We can promptly see that for positions above $18 \mathrm{~cm}$ (z-axis) the density of impurities is indeed negligible.

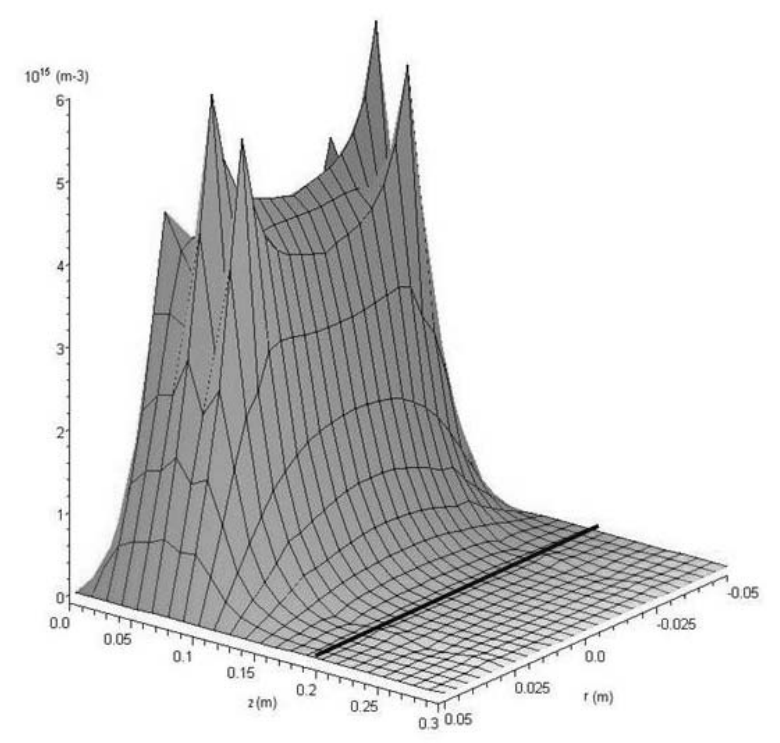

FIG. 3: Density of impurities in the plasma by sputtering on the antenna

\section{EXPERIMENTAL SET-UP}

\subsection{Reactor}

The experimental apparatus consists of a plasma produced by a three loop antenna, placed at the position $\mathrm{z}=3,6$, and $9 \mathrm{~cm}$, inside the vacuum chamber, a stainless steel (316L) cylinder with four ports to allow access of the main diagnostic systems, Langmuir probe, electrostatic energy analyzer, and optical emission spectroscopy (Figure 4).

Usually, the chamber is pumped to a base pressure of $10^{-7}$ mbar and during it operation it is filled with argon to the working pressure, which is kept constant. It has two separated retractile manipulators facing each other, on which the Langmuir and Faraday cup are attached.

\subsection{Oscillator}

The RF power supply is based on a push-pull oscillator, operating at $13.56 \mathrm{MHz}$, with output power up to $600 \mathrm{~W}$. The oscillator uses a 304TL tube and the electrical circuit can be seen in figure 5. The output of the power supply is balanced to produce an inductively coupled plasma. The capacitors $C 9$ and $C 10$ are the decoupling capacitors used to block the DC polarization voltage from the grid of the tube. This voltage can produce capacitive coupling between the RF antenna and the chamber wall, which is grounded. This configuration ensures that the plasma is purely inductively coupled [6]. The $\mathrm{RF}$ frequency is determined by the $C 3$ and $C 4$ variable capac- 


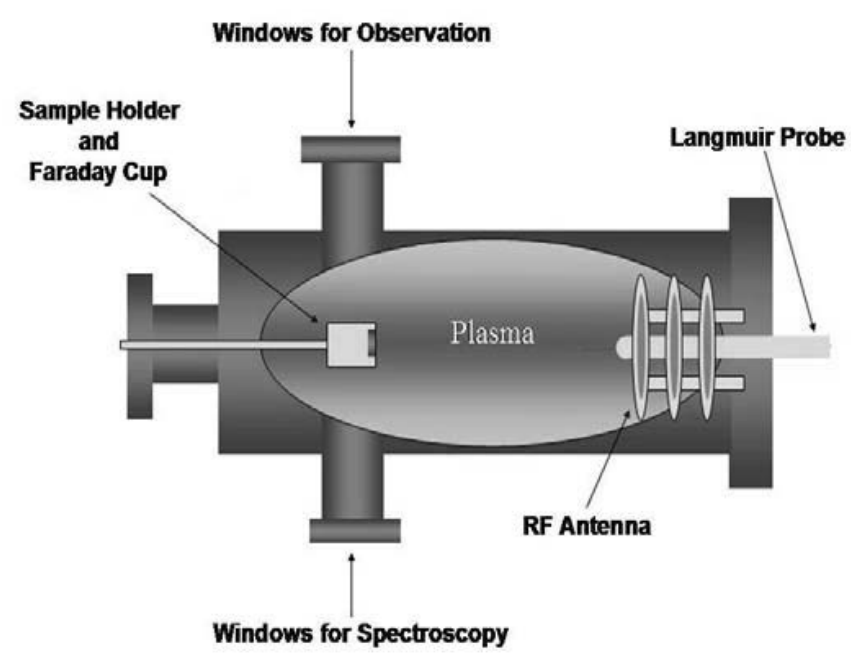

FIG. 4: Schematic drawing of the plasma reactor

itors in tandem configuration.

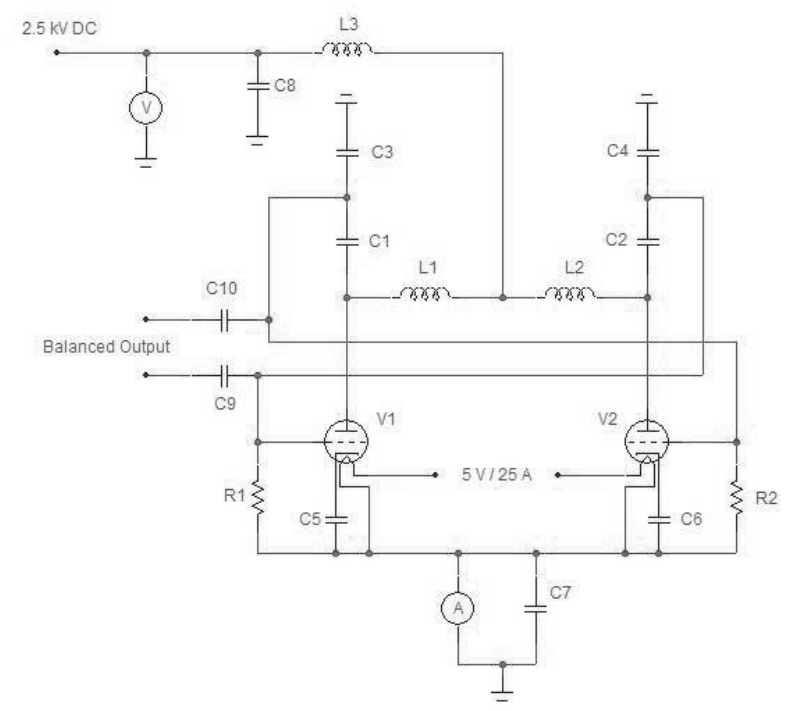

FIG. 5: Electric Circuit of the Push-Pull Oscillator. The values of components in the circuit are: $C 1=C 2=56 \mathrm{pF} / 5 \mathrm{kV} ; C 3=C 4=$ $500 \mathrm{pF} / 1 \mathrm{kV}$ (tandem); $C 5=C 6=C 7=100 \mathrm{pF} / 400 \mathrm{~V} ; C 8=10 \mathrm{nF} /$ $3 \mathrm{kV} ; C 9=C 10=470 \mathrm{nF} / 1 \mathrm{kV} ; R 1=R 2=2.5 \mathrm{k} \Omega ; L 1=L 2=7 \mu \mathrm{H}$ and $L 3=1 \mathrm{mH}$

The 304TL tube is a triode of low amplification factor $(\mu \approx 12)$ with maximum power dissipation on the plate of 300 Watts. The maximum DC voltage on the plate is $3 \mathrm{kV}$ and it can operate at frequencies up to $40 \mathrm{MHz}$.

\subsection{Diagnostic}

The Langmuir probe was constructed with a tungsten tip of $0.5 \mathrm{~mm}$ diameter and $2 \mathrm{~mm}$ length brazed to a glass tube head and connected to a low-pass filter placed inside the tube and close to the probe tip to block the RF fundamental frequency (13.56 MHz) and harmonics. The probe data was obtained using the Druyvesteyn second derivative method; it gives the energy distribution function of particles in the plasma from the second derivative of the Langmuir $I \times V$ curve. The electron energy distribution function, EEDF, is given by

$$
g_{e}(V)=\frac{2 m}{e^{2} A}\left(\frac{2 e V}{m}\right)^{\frac{1}{2}} \frac{d^{2} I}{d V^{2}},
$$

where $e$ and $m$ are the electron charge and mass, respectively, $A$ the probe area, $V=\Phi_{p}-V_{b}$ is the difference between the plasma potential $\Phi_{p}$ and the voltage applied to the Langmuir probe $V_{b}$, and $I$ is the current from the Langmuir probe measured in a shunt resistor.

Once the EEDF is obtained, the density $n_{e}$ can be promptly calculated,

$$
n_{e}=\int_{0}^{\infty} g_{e}(V) d V
$$

and also the effective temperature, given in electron-volts, by

$$
T_{e f f}=\frac{2}{3 n_{e}} \int_{0}^{\infty} V g_{e}(V) d V .
$$

\section{RESULTS AND DISCUSSION}

\subsection{Plasma characterization}

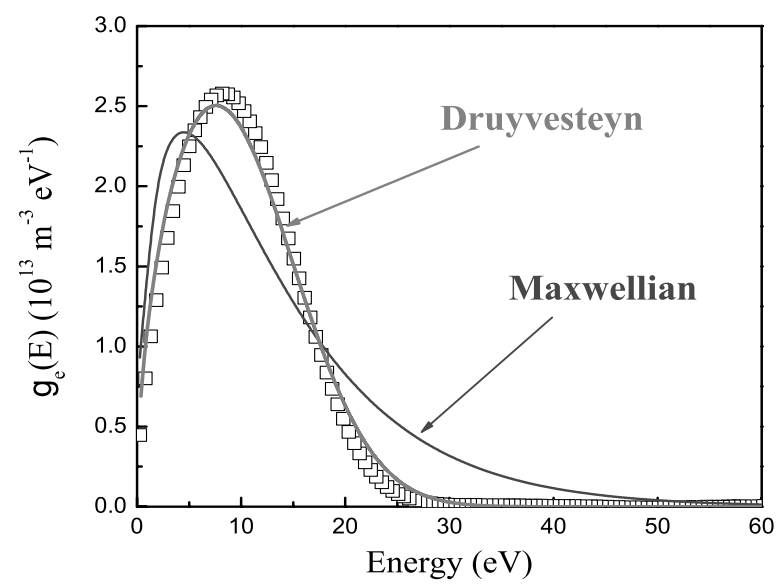

FIG. 6: Electron Energy Distribution Function in $\mathrm{z}=22 \mathrm{~cm}$ and $\mathrm{r}=0 \mathrm{~cm}$ for $\mathrm{p}=3.0 \times 10^{-2} \mathrm{mbar}$ and $\mathrm{P}=120 \mathrm{~W}$

Variations in the density or in the average energy of the species present in the plasma have influence on film deposition, cleaning, and surface treatments. Therefore, knowledge of how external conditions affect intrinsic parameters of the plasma provides a better understanding of the physical mechanisms involved in the process and, therefore, aids to determine the best experimental plasma cleaning condition.

The EEDF was determined for the conditions used for cleaning the sample. The result is given by the dotted curve in figure 6. From this figure, it is quite clear that the EEDF cannot be described by a Maxwellian

$$
f_{M}(W)=\frac{2 n}{\pi^{\frac{1}{2}}} \frac{W^{\frac{1}{2}}}{T_{e}^{\frac{3}{2}}} \exp \left[-\frac{W}{T_{e}}\right] .
$$




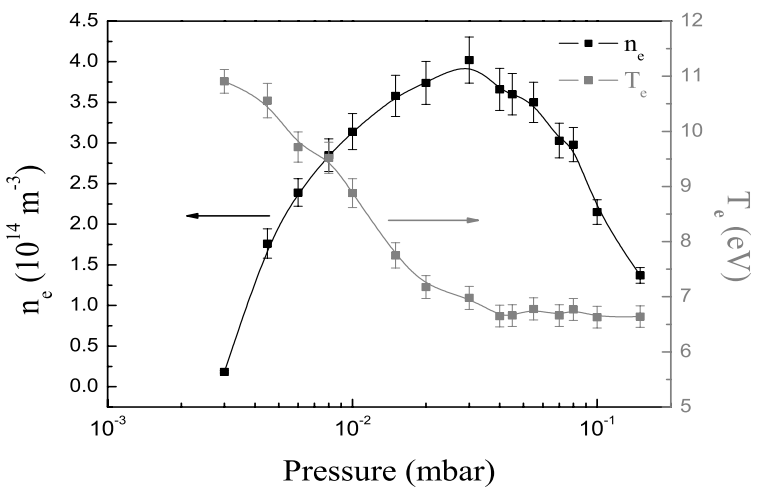

FIG. 7: Electron temperature and density as function of pressure at $\mathrm{r}=0 \mathrm{~cm}$ and $\mathrm{z}=22 \mathrm{~cm}$ for $\mathrm{P}=120 \mathrm{~W}$

However, using the Druyvesteyn energy distribution function, given by

$$
f_{D}(W)=1,04 n \frac{W^{\frac{1}{2}}}{W_{a v}^{\frac{3}{2}}} \exp \left[-\frac{0,55 W^{2}}{W_{a v}^{2}}\right],
$$

and fitting the experimental data, we obtain a quite good agreement.

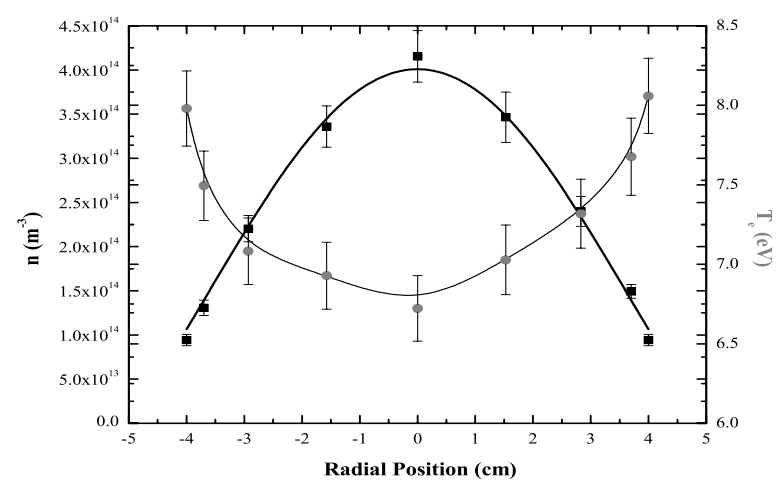

FIG. 8: Electron temperature and density as function of the radial position for $\mathrm{p}=3.0 \times 10^{-2} \mathrm{mbar}$ and $\mathrm{P}=120 \mathrm{~W}$

Using equations 31 and 32, we find the electron temperature and density for several positions inside of the reactor and for several pressures in order to set the best experimental conditions.

To find the pressure that maximizes the plasma density we vary it from $3.0 \times 10^{-3}$ up to $1.5 \times 10^{-1}$ mbar. It turns out that the plasma density presents a maximum value around $p=$ $3.0 \times 10^{-2}$ mbar, as shown in the figure 7 . All measurements were performed in the position of $\mathrm{z}=22 \mathrm{~cm}$, because this is the position in front of the window of observation.

The probe tip is designed so that the radial density and temperature profiles can be obtained by rotating it around the axis [9]. The result is shown in figure 8. While the density has the expected behavior, the electron temperature increases with radius, indicating primary electron energy loss due to impact ionization.

We also characterize the behavior of the plasma along the $\mathrm{z}$ position. As shown in figure 9, the density increases towards the RF antenna, as expected.

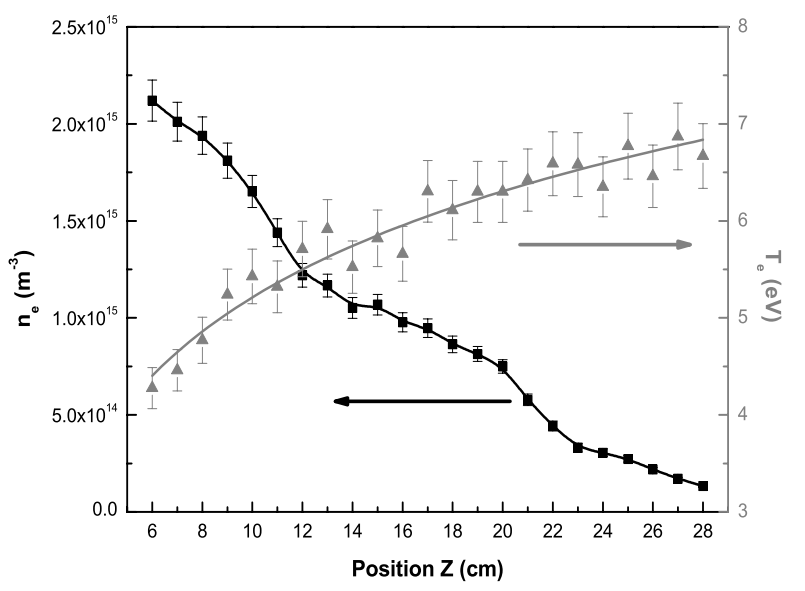

FIG. 9: Electron temperature and density as function of the $\mathrm{z}$ position in $\mathrm{r}=0 \mathrm{~cm}$ for $\mathrm{p}=3.0 \times 10^{-2} \mathrm{mbar}$ and $\mathrm{P}=120 \mathrm{~W}$

\subsection{Cleaning operation}

For the characterization of the device as a plasma cleaner, the Energy Dispersive Spectroscopy (EDS) technique was applied to a titanium sample before and after being exposed to the plasma.

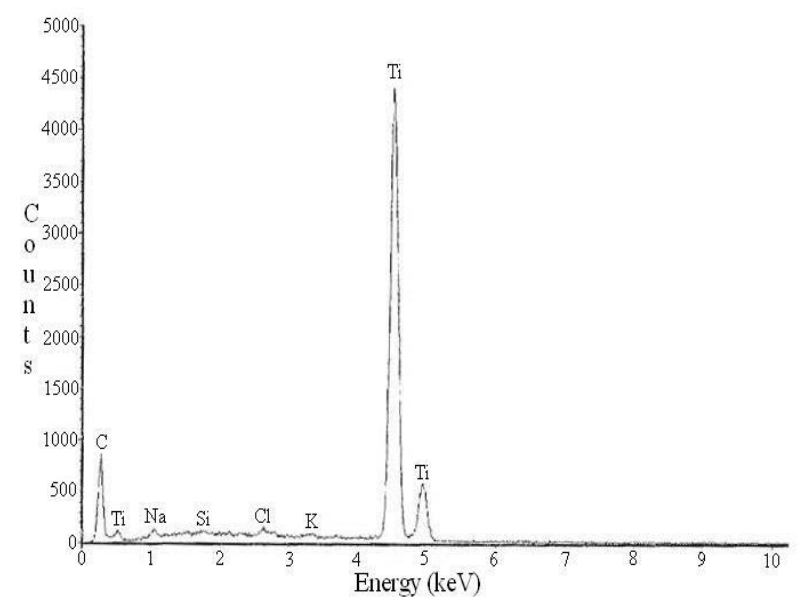

FIG. 10: EDS Spectrum of the Dirty Sample

First, we manipulated the sample carelessly in order to add on impurities to its surface. As can be seen in figure 10, the introduced contaminants are mainly hydrocarbons and salts, i.e., chlorine, sodium, potassium. The silicon peak is due to the presence of silicon oxide in the sample.

Then we treated the sample in the plasma cleaner for 30 minutes with $\mathrm{P}=120 \mathrm{~W}$ RF power, as shown in figure 11 , after exposure, the contaminants were totally removed from the sample. 


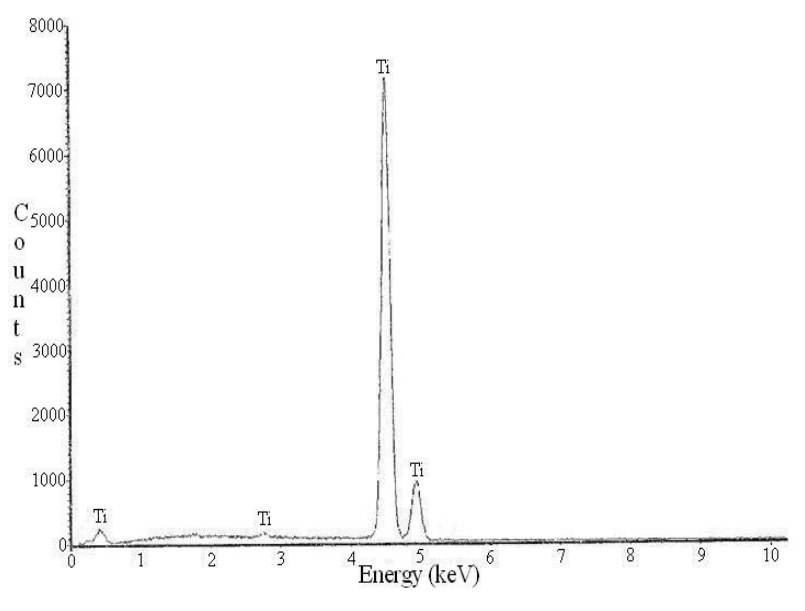

FIG. 11: EDS Spectrum of the Clean Sample

\section{CONCLUSION}

We have described in detail the characterization of a RF plasma device that can be useful for plasma cleaning and surface treatment, while allowing full access for basic plasma studies. It operates intrinsically in the inductive-coupled mode. For the pressure conditions of this work, the EEDF is Druyvesteyn-like. But the device can be readily used to exploit a broad range of operating conditions.

\section{ACKNOWLEDGMENTS}

This work was partially supported by the Brazilian National Council for Research and Development (CNPq). GPC has carried out his work under graduate fellowship from CAPES.
[1] C.O. Jung, K.K. Chu, B.G. Hwang, J.T. Moon, M.Y. Lee e J.G. Lee, Thin Solid Films 341 (1999) p. 112-119.

[2] T.C. Isabell, P.E. Fischione, C.O'Keefe, M.U. Guruz e V.P. Dravid, Microscopy and Microanalysis 5 (1999) p. 126-135.

[3] M.S. Kabir, R.E. Morjan, O.A. Nerushev, P. Lundren, S. Bengtsson, P. Erickson e E.E.B. Campbell, Nanotechnology 16 (2005) p. $458-466$.

[4] K. Ostrikov, Reviews of Modern Physics 77 (2005) p. 489-511.

[5] M. Yamashita, The Journal of Vacuum Science and Technology A 7 (1989) p. 151-158.
[6] G.P. Canal, H. Luna, R.M.O. Galvão, Journal Physics D: Applied Physics 43 (2010) 025209.

[7] M.A. Lieberman e A.J. Lichenberg, Principles of Plasma Discharges and Materials Processing, Wiley, New York 1994.

[8] J.D. Jackson, Classical Electrodynamics - Third Edition, Wiley, Berkeley 1999.

[9] Gustavo Paganini Canal - Master Degree Dissertation, Brazilian Center for Physics Research, Rio de Janeiro (2009). 